\title{
Going flat: examining heterogeneity in the soda-obesity relationship by subgroup and place of birth among Asian Americans
}

\author{
Héctor E Alcalál,* and Mienah Z Sharif ${ }^{2}$ \\ 'Department of Public Health Sciences, University of Virginia, Fontaine Research Park, 560 Ray C. \\ Hunt Drive, Charlottesville, VA 22903, USA: ${ }^{2}$ Department of Medicine, University of California Irvine, Irvine, \\ CA, USA
}

Submitted 1 April 2016: Final revision received 24 November 2016: Accepted 13 January 2017: First published online 24 February 2017

\begin{abstract}
Objective: To determine if the association between soda consumption and obesity is uniform among Asian-American population subgroups.

Design: We conducted multivariate logistic regression analyses on odds of being obese among seven Asian subgroups and by place of birth using data from the 2009 California Health Interview Survey.

Setting: An omnibus population-based health survey.

Subjects: Non-institutionalized adults, aged 18 years or over, residing in California (n 36 271).

Results: Despite low levels of soda consumption in several Asian-American ethnic groups, soda consumption increased the odds of being obese among Chinese, Koreans and Other Asians but not for Whites. Obesity risk varied across Asian subgroups and by place of birth within these subgroups.

Conclusions: More public health efforts addressing soda consumption in AsianAmerican communities are needed as a strategy for not only preventing chronic diseases but also disparities, considering the varying levels of soda intake across subgroups. Results support the growing body of literature critiquing acculturation theory in immigrant health research by documenting inconsistent findings by place of birth. Future research should take into account the heterogeneity among Asian Americans to advance our understanding of health outcomes and disparities.
\end{abstract}

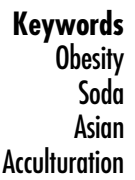

Obesity is a critical public health problem in the USA ${ }^{(1-5)}$. Trends have documented increases in obesity levels across all racial and ethnic groups in the last few decades ${ }^{(2,4)}$. Over a third $(35.7 \%)$ of the adult population is now considered obese ${ }^{(2)}$ and obesity is associated with a wide range of adverse consequences ranging from low self-esteem $^{(6)}$ to type 2 diabetes, heart disease and some cancers $^{(1,7)}$.

The aetiology of obesity is multifaceted and comprises a complex web of factors ${ }^{(1)}$. However, much of the public health efforts have focused on improving the two primary components of energy balance, dietary intake and physical activity $^{(8)}$. In recent years there has been burgeoning interest in the role of sugar-sweetened beverages (SSB) as a critical factor in the obesity epidemic ${ }^{(9-11)}$ considering they are the main source of added sugar in the US $\operatorname{diet}^{(9,12)}$. SSB (e.g. sodas, fruit juices and energy drinks) with energy-containing sweeteners, such as sucrose, highfructose corn syrup or added fruit juice concentrates, are characterized as 'liquid candy' and are considered risk factors for obesity, having replaced more nutritious beverages including milk, $100 \%$ fruit juice and water ${ }^{(8,13)}$. Also, studies have shown that drinking soda, for example, is associated with increased consumption of energy-dense snacks, particularly sweet food items, as researchers posit that the sugary drink increases cravings for sweet food ${ }^{(14)}$. Additionally, people do not report feeling as full as they would have if they consumed the same amount of energy through food and are therefore less likely to compensate their food intake to account for the energy consumed through the beverage ${ }^{(9,15)}$.

Racial/ethnic disparities in obesity have been widely documented $^{(3)}$ with Latinos and Blacks having higher rates than Whites ${ }^{(2,4)}$. Yet, there is a dearth of research looking at disparities in obesity risk via SSB consumption. A limited number of studies have assessed how SSB consumption varies by sociodemographic characteristics ${ }^{(7,16)}$ and found that rates are higher among Blacks, Latinos ${ }^{(17)}$ and people 
who have lower income and lower levels of education ${ }^{(8,16)}$. The present study aims to help fill the gap in the literature by focusing on SSB consumption and obesity levels among Asian Americans, an understudied group in obesity research. Studies are calling for more research on obesity risk factors among Asians ${ }^{(18-22)}$ because, although Asian Americans have lower obesity rates than other racial/ethnic groups $^{(20,23)}$, rates have been increasing over time ${ }^{(19,21)}$. Moreover, Asians constitute the fastest-growing racial group in the USA and the largest proportion of recent immigrants $^{(24)}$ and findings suggest that Asians may be susceptible to obesity-related chronic diseases at lower BMI levels than Whites ${ }^{(5,20)}$.

An existing limitation in public health research among Asian Americans is the tendency to aggregate data such that they are analysed and discussed as one group (collapsing a heterogeneous group including East Asian Americans and South Asian Americans or US-born and foreign-born Asians). This approach disguises disparities between and within groups that other research has noted $^{(18,21,25,26)}$.

Given the large proportion of immigrants among Asian Americans, we were motivated by studies critiquing the overemphasis on negative acculturation effect, which posits that immigrants come to the USA with superior health behaviours that then worsen over time and among subsequent generations, in immigrant health research ${ }^{(22)}$. Specifically, we attempted to illustrate that there are indeed mixed findings not only by Asian-American subgroups in general, but also by place of birth, to help build the growing body of literature challenging the conventional approach of acculturation theory ${ }^{(22,27)}$. Thus, the purpose of the current study was to provide preliminary analyses examining the association between SSB (specifically soda) consumption and obesity risk among various Asian-American subgroups and by place of birth. The hope was to determine if the deleterious effects of soda are uniform across groups and if they vary by place of birth for individuals of the same Asian-American subgroup.

\section{Methods}

\section{Data source}

The present study used data from the 2009 Adult California Health Interview Survey (CHIS), a cross-sectional survey of California adults (aged 18 years or over) conducted between September 2009 and April 2010. The CHIS used a multistage sample design aimed to be representative of the household-dwelling California adult population as well as to provide estimates for the state's major racial and ethnic subgroups. Using random digit dialling, households were contacted and a random adult within the household was selected to participate in the CHIS. The CHIS was administered in English, Spanish, Mandarin, Cantonese, Vietnamese and Korean using a computer-assisted telephone interviewing system. The CHIS includes replicate weights and adjustments to account for differential selection probabilities, non-response bias and stratification ${ }^{(28)}$. CHIS data used in the present study were de-identified and publicly available, thus not requiring approval by an institutional review board. The original study was conducted with institutional review board approval through the University of California, Los Angeles and using verbal consent.

While more recent versions of the CHIS have been conducted, CHIS 2009 was selected because it included a targeted oversample of Korean-American and VietnameseAmerican individuals, which was not conducted in subsequent years ${ }^{(28)}$.

Overall, 47614 adults completed the survey and missing data were imputed using hot deck imputation by the $\mathrm{CHIS}^{(28)}$. For the present study the sample was restricted to the 36271 respondents who identified as non-Latino White or Asian. In all, 31167 respondents were non-Latino White and 5104 were Asian American (specifically 1065 Chinese, 428 Japanese, 958 Korean, 507 Filipino, 420 South Asian, 1423 Vietnamese, and 303 were from other Asian-American subgroups).

\section{Variables}

The independent variable of interest was soda consumption. Respondents were asked: 'During the past month, how often did you drink soda or pop that contains sugar? Do not include diet soda.' Respondents indicated how many times in the past month they drank soda. For the current study, a CHIS-constructed variable that converted this to times per week, by dividing the month value by 30 to convert to times for day and multiplying by 7 , was used. The CHIS flagged, removed and imputed seven cases that had daily soda consumption of eight or more times ${ }^{(29)}$.

The main predictors of interest were Asian-American identity and place of birth. Asian-American identity was broken down into seven national- and regional-origin subgroups: Chinese, Japanese, Korean, Filipino, South Asian, Vietnamese and Other Asian. This latter category included individuals who identified as South-East Asians or with two or more Asian subgroups. For some analyses, individuals identifying as non-Latino White were used as the reference group. Place of birth was measured as US-born $v$. foreign-born, with US-born individuals serving as the reference group.

The main outcome of interest was obesity (i.e. BMI greater than or equal to $\left.30 \mathrm{~kg} / \mathrm{m}^{2}\right)^{(30)}$. Gender, age, income and years of education were included as control variables. Males served as the reference group for gender. Age was measured continuously, but all ages above 85 years were top coded to age 85 years in the CHIS. Income was measured as annual household income in thousands of US dollars. The CHIS top coded all responses greater than $\$$ US 300000 , as $\$$ US 300000 . Years of education were originally coded into broad categories: no formal 
education, grade 1-8, grade 9-11, grade 12/high school, some college, vocational school, AA/AS, BA/BS, some grad school, MA/MS, PhD or equivalent. These categories were recoded to represent years of education completed by taking the year values in the middle of each category or the year equivalent.

\section{Statistical analysis}

All analyses were conducted using the statistical software package Stata version 14.1, using eighty replicate weights. First, descriptive statistics were run (i.e. means and frequencies), stratified by race (Table 1 ). These descriptive statistics where then compared by Asian-American subgroup using linear and logistic regression (Table 1). Next, a logistic regression model predicting odds of obesity from Asian subgroups, soda consumption and race by soda interaction terms was run (Table 2). This model controlled for gender, age, years of education and income. Finally, a model for each subgroup was run predicting obesity from nativity status, while controlling for gender, age, years of education and income (Table 3). For logistic regression analyses in Tables 2 and 3, models were rerun by altering the reference groups for ethnic groups and place of birth so as to provide a complete picture of interactions. To evaluate model fit, the Hosmer-Lemeshow Goodness of Fit (HLGOF) statistic was calculated for logistic regression models. Finally, to illustrate interactions, predicted probabilities of obesity were plotted by Asian-American subgroup, mirroring the results from Table 2 .

\section{Results}

As Table 1 shows, less than a fifth of the sample was obese, with non-Latino Whites having the highest rates of obesity (21.08\%). Among the subgroups, Japanese Americans had the highest rates of obesity (13.73\%) and Chinese Americans had the lowest (3.72\%). Overall, respondents reported drinking soda, on average, 1.67 times per week. Among Asian Americans, this value was highest among Filipino Americans and lowest among Chinese Americans (1.81 v. 0.68). Among the sample overall, $22.61 \%$ was foreign-born with South Asian Americans having the highest (84.76\%) and Japanese Americans having the lowest rate (21.32\%). Overall, the average age was 47.56 years, the average level of education was a little more than an AA/AS degree (14.41 years) and the average household income was \$US 88610 in the past year. Slightly more than half of the sample was female $(51.45 \%)$. All descriptive statistics varied by subgroup $(P<0.05)$.

\section{Relationship between soda consumption and odds} of being obese by Asian subgroup

Table 2 shows a logistic regression model predicting obesity from race, obesity from soda consumption and the

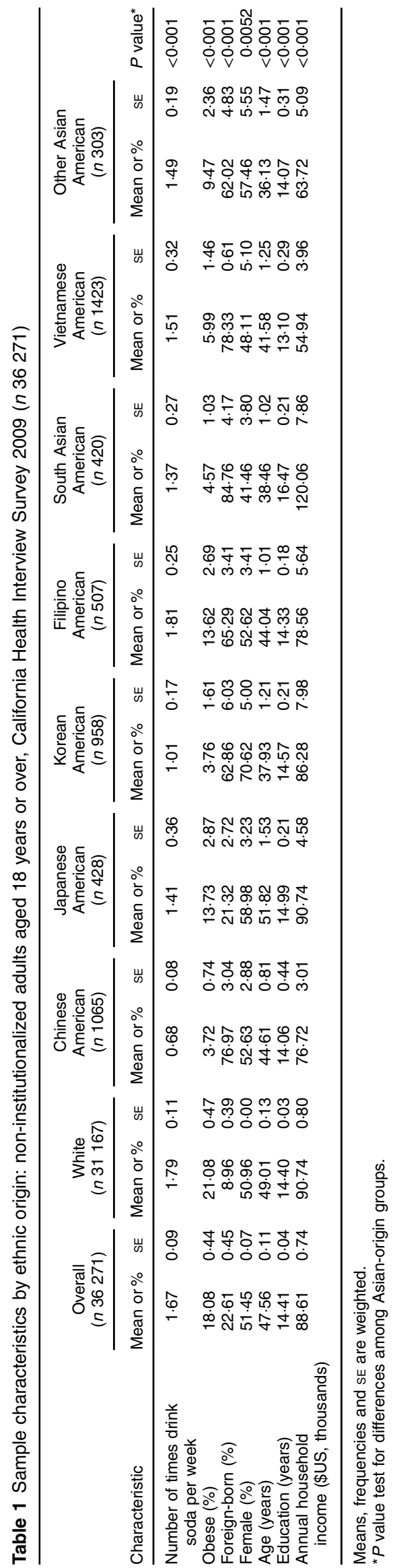


Table 2 Logistic regression predicting obesity from soda consumption and ethnicity among non-institutionalized adults aged 18 years or over, California Health Interview Survey 2009 (n 36 271)

\begin{tabular}{|c|c|c|}
\hline Variable & $\mathrm{AOR}^{*}$ & $95 \% \mathrm{Cl}$ \\
\hline Number of times drink soda per week $\dagger$ & 1.00 & $0.99,1.02$ \\
\hline \multicolumn{3}{|l|}{ Ethnicity $\ddagger$} \\
\hline White & \multicolumn{2}{|c|}{ Ref. } \\
\hline Chinese American & 0.11 & $0.07,0.17$ \\
\hline Japanese American & 0.58 & $0.35,0.94$ \\
\hline Korean American & 0.11 & $0.06,0.22$ \\
\hline Filipino American & 0.62 & $0.38,1.02$ \\
\hline South Asian American & 0.22 & $0.14,0.33$ \\
\hline Vietnamese American & 0.21 & $0.13,0.35$ \\
\hline Other Asian American & 0.27 & $0.12,0.61$ \\
\hline \multicolumn{3}{|l|}{ Asian heritage group by soda interaction§ } \\
\hline Chinese American $\times$ soda & 1.22 & $1.09,1.37$ \\
\hline Japanese American $\times$ soda & 1.04 & $0.95,1.14$ \\
\hline Korean American $\times$ soda & 1.22 & $1.01,1.46$ \\
\hline Filipino American $\times$ soda & 0.97 & $0.86,1.09$ \\
\hline South Asian American $\times$ soda & 1.00 & $0.88,1.14$ \\
\hline Vietnamese American $\times$ soda & 0.99 & $0.88,1.13$ \\
\hline Other Asian American × soda & 1.19 & $1.03,1.38$ \\
\hline \multicolumn{3}{|l|}{ Sociodemographic characteristics } \\
\hline \multicolumn{3}{|l|}{ Gender } \\
\hline Male & \multicolumn{2}{|c|}{ Ref. } \\
\hline Female & 0.73 & $0.66,0.80$ \\
\hline Age (years) & 1.01 & $1.00,1.01$ \\
\hline Years of education & 0.94 & $0.92,0.97$ \\
\hline \multirow[t]{2}{*}{ Annual household income (\$US, thousands) } & 1.00 & $1.00,1.00$ \\
\hline & $F$ & $P$ value \\
\hline Test for interaction & $2 \cdot 82$ & 0.0118 \\
\hline Hosmer-Lemeshow Goodness of Fit statistic & 0.47 & 0.8868 \\
\hline
\end{tabular}

AOR, adjusted OR; ref., reference category.

Significant AOR and $95 \% \mathrm{Cl}$ denoted in bold.

${ }^{*}$ Adjusted for gender, age, years of education and income.

†Represents the effect of each additional soda consumed for Whites.

$\ddagger$ Represents the odds of being obese for each ethnic-origin group, relative to

Whites and soda consumption of zero times per week.

$\S$ Represents the difference in the effect of each additional soda consumed for Asian-American subgroup, relative to Whites.

race by soda consumption interaction, while controlling for demographic characteristics. With the exception of Filipino Americans, all subgroups had lower odds of obesity relative to non-Latino Whites when soda consumption was equal to zero $(P<0.05)$. The soda consumption term in the model reveals that soda consumption was not associated with odds of obesity among non-Latino Whites (adjusted OR (AOR) $=1.00 ; 95 \%$ CI 0.99, 1.02). However, each additional time soda was consumed in a week was associated with increased odds of obesity among Chinese Americans (AOR $=1 \cdot 22 ; 95 \%$ CI 1.09 , 1.37), Korean Americans ( $\mathrm{AOR}=1 \cdot 21 ; 95 \%$ CI 1.02, 1.46) and Other Asian Americans (AOR $=1 \cdot 19 ; 95 \%$ CI 1.04, 1.39; these AOR are not shown in Table 2). Overall, there was a significant interaction between soda consumption and race/ethnicity $(P=0 \cdot 0118)$. Specifically, soda consumption had a larger obesogenic impact among ChineseAmerican, Korean-American and Other Asian-American groups, relative to non-Latino Whites $(P<0.05)$. Female gender $(\mathrm{AOR}=0.73 ; 95 \% \mathrm{CI} 0 \cdot 66,0 \cdot 80)$, each additional year of education $(\mathrm{AOR}=0.94 ; 95 \% \mathrm{CI} 0.92,0.97)$ and each additional thousand US dollars in annual household income $(\mathrm{AOR}=1 \cdot 00 ; 95 \% \mathrm{CI} 1 \cdot 00,1 \cdot 00)$ were associated with lower odds of obesity. The HLGOF statistic showed that the model fit the data reasonably well $(P=0 \cdot 89)$.

Figure 1 visually depicts the findings from analyses shown in Table 2. Here we see that the predicted probability of obesity is fairly constant for most ethnic groups, irrespective of soda consumption. However, predicted probability of obesity increases with soda consumption for Chinese Americans, Korean Americans and Other Asian Americans.

\section{Relationship between soda consumption and odds of being obese by Asian subgroup and place of birth} Table 3 shows stratified logistic regression models predicting obesity from place of birth, obesity from soda consumption and the place of birth by soda consumption interaction, while controlling for demographic characteristics. Foreign-born Chinese Americans and Korean Americans had lower odds of obesity than their US-born counterparts when soda consumption was equal to zero $(P<0.05)$. The soda consumption term in each model reveals that, among individuals born in the USA, each additional time soda was consumed in a week was associated with increased odds of obesity among Vietnamese Americans (AOR =1.22; $95 \%$ CI 1.04, 1.44) and Other Asian Americans (AOR =1.44; 95\% CI 1.18, 1.76). Among individuals born outside the USA, each additional time soda was consumed in a week was associated with increased odds of obesity among Chinese Americans (AOR $=1.27$; $95 \%$ CI 1.08, 1.48), Japanese Americans (AOR $=1.15$; $95 \%$ CI 1.06, 1.25) and Korean Americans (AOR =1.81; 95\% CI 1.20, 2.74; these AOR are not shown in Table 3 ). There was a significant interaction between place of birth and soda consumption for Japanese Americans, Vietnamese Americans and Other Asian Americans $(P<0 \cdot 05)$. Specifically, there was a greater impact of soda consumption on the odds of being obese among foreign-born Japanese Americans in comparison to their US-born peers. Conversely, there was a smaller impact of soda consumption on obesity risk among foreign-born Vietnamese Americans and Other Asian Americans, relative to each group's US-born counterparts. Across models, sociodemographic characteristics were not consistently associated with odds of being obese. Overall, the HLGOF statistics suggested that model fit was poor.

\section{Discussion}

In recent years, there has been growing interest in reducing consumption of SSB, or "empty liquid calories'(14), as a strategy for reducing obesity levels. The current study is one of the first, to the authors' knowledge, that examines soda consumption among Asian-American subgroups and by place of birth in California, the state with the largest proportion of immigrants ${ }^{(24)}$. While we examined other SSB 


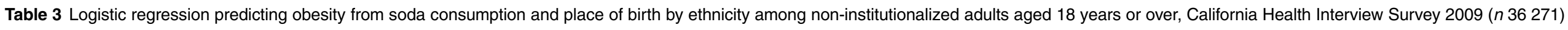

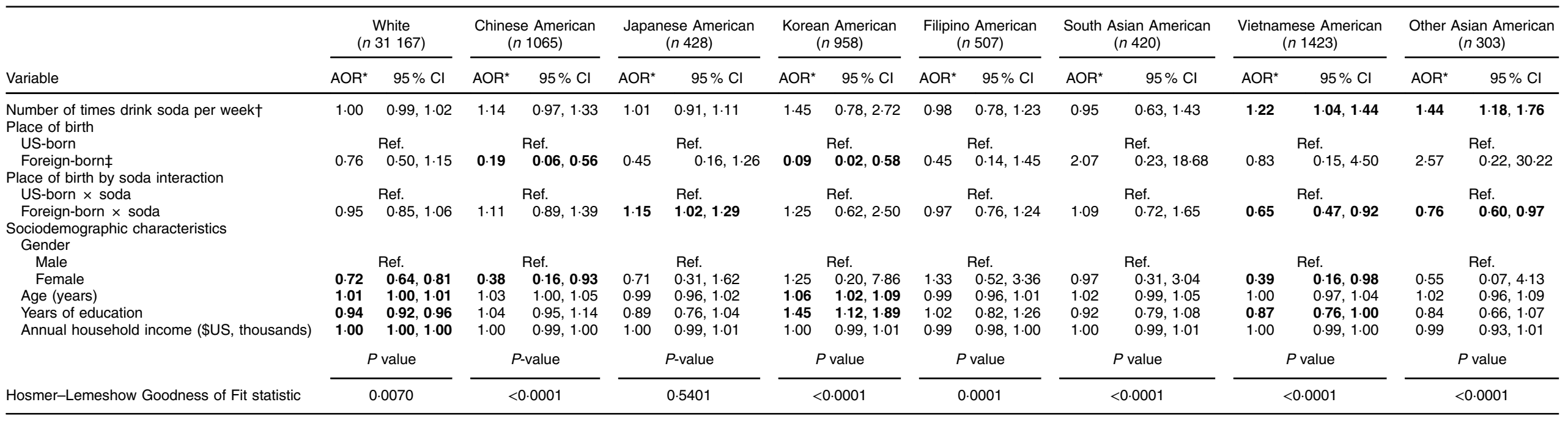

\section{AOR, adjusted OR; ref., reference category.}

'Significant $\mathrm{AOR}$ and $95 \% \mathrm{Cl}$ denoted in bold.

†Represents the effect of each additional soda consumed for US-born individuals.

†Represents the odds of being obese for foreign-born individuals, relative to US-born individuals and soda consumption of zero times per week. 


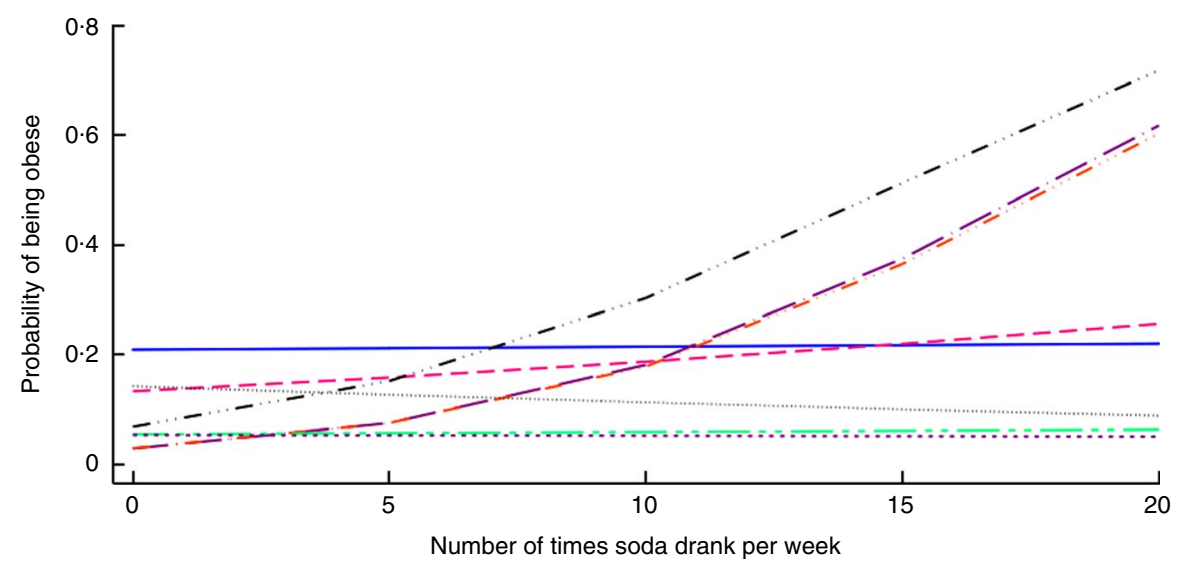

Fig. 1 (colour online) Predicted probability of obesity according to soda consumption and ethnicity (- White; ----, Japanese American; ..........., Filipino American; ........, Vietnamese American; -._.., Chinese American; -......., Korean American; -.--., South Asian American; -......-., Other Asian American) among non-institutionalized adults aged 18 years or over, California Health Interview Survey 2009 (n 36 271)

(i.e. energy/sports drinks and fruit-flavoured drinks), there were no significant interactions between Asian-American subgroups and SSB consumption when compared with Whites, suggesting that this relationship may be unique to soda.

Our findings are consistent with published literature, albeit limited, that obesity levels are lower among Asian Americans in comparison to Whites ${ }^{(19,21,26,31)}$. However, the disaggregation of the Asian-American category into national- and regional-origin subgroups helped elucidate the heterogeneity and disparities across groups. Namely, we found that Japanese-American respondents had the highest rates of obesity whereas Chinese Americans had the lowest. These findings, in conjunction with trends documenting increasing rates of obesity among Asian Americans ${ }^{(21,32)}$, emphasize the need for more obesity prevention efforts in these communities and the need to better understand and address the differences in health behaviours and trends within these groups ${ }^{(19,21,31)}$. While there are a number of potential factors driving differences between Asian Americans (i.e. fraction of foreign-born, obesity rates in home country, inhabiting enclaves in the USA and socio-economic differences), these effects cannot be teased apart in the present study because we do not know enough about the immigration experiences of respondents or the social and physical environments that they occupy in the USA.

With regard to soda intake, we found that levels are generally low and that all Asian-American subgroups, with the exception of Filipinos, reported drinking soda less often than Whites. This finding is consistent with a prior study that disaggregated Asian-American subgroups and found that Filipinos had a higher rate of engaging in obesityrelated dietary risk factors than other subgroups ${ }^{(19,21,31)}$. That study ${ }^{(19,21,31)}$, however, focused on adolescents. Thus, the current study adds to the literature on obesity and disparities by focusing on variations in soda intake and across subgroups among Asian-American adults.
When we extended the analyses to look at this relationship in terms of the odds of being obese, our study highlighted the unique role soda may play in increasing disparities in obesity rates in Asian-American communities. Namely, the results demonstrated that for each additional time soda was consumed, there was an increased likelihood of being obese among some subgroups; a relationship that did not hold true among Whites. These results suggest that efforts to decrease soda consumption among Asian Americans may have more of an effect in reducing obesity rates than among Whites. Or stated differently, the lack of attention on soda consumption among Asian Americans is a missed opportunity to reduce current and prevent future increased levels of obesity. Moreover, the disaggregation of subgroups elucidated interesting differences. Namely, soda consumption among Chinese and Korean and Other Asian Americans had more of an impact on the odds of being obese, relative to Whites. These findings extend previous studies that found varying health behaviours across Asian-American subgroups ${ }^{(19,20)}$ by showing that behaviours can also have different impacts on health outcomes across subgroups.

Considering that Asians comprise the fastest-growing group of immigrants, another focus of our study was to delineate the effects of this relationship by respondent's place of birth. Our findings, similar to other studies ${ }^{(19-22)}$, support the growing idea that immigrant health research must recognize that patterns are far from yielding a clear and consistent story ${ }^{(22,33)}$. For example, the odds of obesity were lower among foreign-born Chinese Americans and Korean Americans, relative to their US-born peers. This finding is supported by the negative acculturation effect positing that health behaviours and outcomes are better among foreign-born immigrants and deteriorate among subsequent generations including those born in the USA. However, the impact of soda consumption on obesity complicates this picture. Namely, soda consumption had a 
diminished obesogenic impact among foreign-born Vietnamese Americans and Other Asian Americans, relative to US-born respondents from those subgroups, while being foreign-born had an increased obsesogenic impact, relative to their US-born peers. In other words, the results support the call for a research agenda on immigrant health that recognizes the diverse cultural and political contexts immigrants originate from, as well as the heterogeneity of socio-economic and health profiles among immigrants from the same sending country that may help explain variation in health behaviours and health outcomes ${ }^{(22,25,33,34)}$.

These mixed findings are consistent with other studies and serve as preliminary analyses on a topic that merits future research efforts. Moreover, the lack of statistically significant differences within most of the subgroups by place of birth emphasizes the need for a more comprehensive approach towards studying immigrant health that takes into consideration trends in the sending contexts. For example, the nutrition transition, in which people are already adopting what is characterized as the 'Western lifestyle' within their own countries pre-migration ${ }^{(22)}$, is one of many factors that may help explain the lack of consistent findings on dietary behaviours and obesity risk among groups in the USA.

\section{Limitations}

A limitation of the current study is the main measures of interest. Namely, soda intake and obesity are based on self-reported measures that may be biased. Also, the soda measure does not capture the servings consumed, thereby undermining our ability to ascertain the actual energy content being consumed. However, the authors used this measure as has been done in published studies ${ }^{(7,17)}$. In addition, the current study is cross-sectional and therefore cannot infer causality between the two main variables of interest. Moreover, one may also argue that the relationship is one of reverse causality, namely that obesity increases one's likelihood of drinking more soda. Although the study advances the literature on obesity risk factors among an understudied population, CHIS does not provide subgroup information for all Asian ethnicities or subgroups in their public-use file. Also, some of these groups are too small to analyse independently. An example of this is the 'Other Asian' group, which includes many smaller Asian-American subgroups and those who identify with more than one Asian-American subgroup. As a result, conclusions and generalizations about this group cannot be made. Furthermore, the CHIS does not collect data in all Asian languages, rendering it less representative of some Asian-American subgroups. Also, while the HLGOF has limitations when examining rare outcomes ${ }^{(35)}$ (outcome occurs less than $10 \%$ of the time), the results of the HLGOF test showed that analyses in Table 3 did not fit the data particularly well. As a result, these findings should be interpreted cautiously. Lastly, CHIS is representative of the adult population in California; therefore the authors caution readers from extrapolating the findings to Asian-American subgroups in other states.

\section{Conclusion}

Albeit limited, the current study echoes the mixed findings reported elsewhere when Asian subgroups are disaggregated $^{(19-22)}$. The limited body of knowledge on this topic undermines our ability to contextualize our findings. Therefore it is our hope that the present study will motivate future research on health behaviours and outcomes to further pursue the disaggregation of Asian-American subgroups, as others have called for ${ }^{(36)}$, thereby acknowledging the different experiences, norms, behaviours and socio-economic profiles among immigrant groups ${ }^{(22)}$. These are crucial steps for advancing our understanding and approaches in immigrant and disparities research.

In addition, the findings of the study identify soda consumption as a risk factor that may be contributing to the increasing rates of obesity among some Asian-American subgroups. Moreover, the results identify certain subgroups at a higher risk of obesity via soda consumption. The current study underscores the need for more focused multilevel public health efforts, including health education, social marketing and improving the built environment, to reduce the level of soda consumption in Asian-American communities.

\section{Acknowledgements}

Financial support: This research was made possible by support from the National Center for Advancing Translational Sciences (NIH NCATS; grant number UL1 TR001415). Funders played no role in study design, analyses or interpretation of data. Conflict of interest: Neither author has financial or other conflicts of interests to disclose. Authorship: Both authors contributed equally to this manuscript as follows: H.E.A. led the conceptualization of the study, execution of analyses and writing of results. M.Z.S. led the writing of background and conclusions. Both authors approved this manuscript. Ethics of buman subject participation: This study involved secondary data analyses of de-identified information, thus it did not meet the definition of human subjects research.

\section{References}

1. Chan RS \& Woo J (2010) Prevention of overweight and obesity: how effective is the current public health approach. Int J Environ Res Public Health 7, 765-783. 
2. Flegal KM, Carroll MD, Kit BK et al. (2012) Prevalence of obesity and trends in the distribution of body mass index among US adults, 1999-2010. JAMA 307, 491-497.

3. Kirby JB, Liang L, Chen HJ et al. (2012) Race, place, and obesity: the complex relationships among community racial/ethnic composition, individual race/ethnicity, and obesity in the United States. Am J Public Health $\mathbf{1 0 2}$ 1572-1578.

4. Ogden CL, Carroll MD, Kit BK et al. (2012) Prevalence of obesity and trends in body mass index among US children and adolescents, 1999-2010. JAMA 307, 483-490.

5. Rao G, Powell-Wiley TM, Ancheta I et al. (2015) Identification of obesity and cardiovascular risk in ethnically and racially diverse populations: a scientific statement from the American Heart Association. Circulation 132, 457-472.

6. Clarke PJ, O'Malley PM, Schulenberg JE et al. (2010) Midlife health and socioeconomic consequences of persistent overweight across early adulthood: findings from a national survey of American adults (1986-2008). Am J Epidemiol 172, 540-548.

7. Babey SH, Jones M, Yu H et al. (2009) Bubbling over: soda consumption and its link to obesity in California. Policy Brief UCLA Cent Health Policy Res issue PB-2009-5, 1-8.

8. Woodward-Lopez G, Kao J \& Ritchie L (2011) To what extent have sweetened beverages contributed to the obesity epidemic? Public Health Nutr 14, 499-509.

9. Hu FB \& Malik VS (2010) Sugar-sweetened beverages and risk of obesity and type 2 diabetes: epidemiologic evidence. Physiol Behav 100, 47-54.

10. Sharkey JR, Johnson CM \& Dean WR (2011) Less-healthy eating behaviors have a greater association with a high level of sugar-sweetened beverage consumption among rural adults than among urban adults. Food Nutr Res 2011, 55.

11. Falbe J, Rojas N, Grummon AH et al. (2015) Higher retail prices of sugar-sweetened beverages 3 months after implementation of an excise tax in Berkeley, California. Am J Public Health 105, 2194-2201.

12. Malik VS, Pan A, Willett WC et al. (2013) Sugar-sweetened beverages and weight gain in children and adults: a systematic review and meta-analysis. Am J Clin Nutr 98 , 1084-1102.

13. Nielsen SJ \& Popkin BM (2004) Changes in beverage intake between 1977 and 2001. Am J Prev Med 27, 205-210.

14. Bleich SN, Wolfson JA, Vine S et al. (2014) Diet-beverage consumption and caloric intake among US adults, overall and by body weight. Am J Public Health 104, e72-e78.

15. Harvard School of Public Health (2012) The Problem: Sugary Drinks are a Major Contributor to the Obesity Epidemic. https://www.hsph.harvard.edu/nutritionsource/ sugary-drinks-fact-sheet/ (accessed February 2016).

16. Han E \& Powell LM (2013) Consumption patterns of sugarsweetened beverages in the United States. J Acad Nutr Diet 113, 43-53.

17. Kumar GS, Pan L, Park S et al. (2014) Sugar-sweetened beverage consumption among adults - 18 States, 2012. MMWR Morb Mortal Wkly Rep 63, 686-690.

18. Davis J, Busch J, Hammatt Z et al. (2004) The relationship between ethnicity and obesity in Asian and Pacific Islander populations: a literature review. Ethn Dis 14, 111-118.

19. Guerrero AD, Ponce NA \& Chung PJ (2015) Obesogenic dietary practices of Latino and Asian subgroups of children in California: an analysis of the California Health Interview Survey, 2007-2012. Am J Public Health 105, e105-e112.

20. Maxwell AE, Crespi CM, Alano RE et al. (2012) Health risk behaviors among five Asian American subgroups in California: identifying intervention priorities. J Immigr Minor Health 14, 890-894.

21. Park Y, Wang S, Kitahara CM et al. (2014) Body mass index and risk of death in Asian Americans. Am J Public Health 104, 520-525.

22. Anda RF, Whitfield CL, Felitti VJ et al. (2014) Adverse childhood experiences, alcoholic parents, and later risk of alcoholism and depression. Psychiatr Serv 53, 1001-1009.

23. Bates LM, Acevedo-Garcia D, Alegria M et al. (2008) Immigration and generational trends in body mass index and obesity in the United States: results of the National Latino and Asian American Survey, 2002-2003. Am J Public Health 98, 70-77.

24. Pew Research Center (2013) The Rise of Asian Americans. http://www.pewsocialtrends.org/2012/06/19/the-rise-of-asianamericans/ (accessed January 2017).

25. Holland AT \& Palaniappan LP (2012) Problems with the collection and interpretation of Asian-American health data: omission, aggregation, and extrapolation. Ann Epidemiol 22, 397-405.

26. Wang Y \& Beydoun MA (2007) The obesity epidemic in the United States - gender, age, socioeconomic, racial/ethnic, and geographic characteristics: a systematic review and meta-regression analysis. Epidemiol Rev 29, 6-28.

27. Viruell-Fuentes EA, Miranda PY \& Abdulrahim S (2012) More than culture: structural racism, intersectionality theory, and immigrant health. Soc Sci Med 75, 2099-2106.

28. California Health Interview Survey (2011) Report 1: Sample Design. CHIS 2009 Methodology Series. Los Angeles, CA: California Health Interview Survey.

29. California Health Interview Survey (2011) Dietary Screener in the 2009 CHIS: Scoring Procedures. Los Angeles, CA: California Health Interview Survey.

30. Centers for Disease Control and Prevention (2015) Assessing Your Weight. http://www.cdc.gov/healthyweight/ assessing/bmi/adult_bmi/ (accessed February 2016).

31. Wang S, Quan J, Kanaya AM et al. (2011) Asian Americans and obesity in California: a protective effect of biculturalism. J Immigr Minor Health 13, 276-283.

32. Lu W, Diep CS \& McKyer LJ (2015) Risk factors for childhood obesity among Asian Americans: a systematic review of literature and recommendations for health care research. $J$ Health Care Poor Underserved 26, 171-190.

33. Takeuchi DT, Zane N, Hong S et al. (2007) Immigrationrelated factors and mental disorders among Asian Americans. Am J Public Health 97, 84-90.

34. Gee GC, Ro A, Gavin A et al. (2008) Disentangling the effects of racial and weight discrimination on body mass index and obesity among Asian Americans. Am J Public Health 98, 493-500.

35. Hosmer DW, Lemeshow S \& Klar J (1988) Goodness-of-fit testing for the logistic regression model when the estimated probabilities are small. Biom J 30, 911-924.

36. Chen JY, Diamant AL, Kagawa-Singer M et al. (2004) Disaggregating data on Asian and Pacific Islander women to assess cancer screening. Am J Prev Med 27, 139-145. 